\title{
Potent Sodium/Glucose Cotransporter SGLT1/2 Dual Inhibition Improves Glycemic Control Without Marked Gastrointestinal Adaptation or Colonic Microbiota Changes in Rodents
}

\author{
Fuyong Du, ${ }^{1}$ Simon A. Hinke, ${ }^{1}$ Cassandre Cavanaugh, David Polidori, Nathanial Wallace, \\ Thomas Kirchner, Matthew Jennis, Wensheng Lang, Gee-Hong Kuo, Micheal D. Gaul, \\ James Lenhard, Keith Demarest, Nadim J. Ajami, Yin Liang, and Pamela J. Hornby \\ Cardiovascular and Metabolism Discovery (F.D., S.A.H., C.C., N.W., T.K., M.J., G.-H.K., M.D.G., J.L., K.D., Y.L., P.J.H.) and \\ Analytical Sciences (W.L.), Janssen R\&D LLC, Spring House, Pennsylvania; Cardiovascular and Metabolism Experimental and \\ Translational Medicine, Janssen R\&D LLC, San Diego, California (D.P.); and Alkek Center for Metagenomics and Microbiome \\ Research, Department of Molecular Virology and Microbiology, Baylor College of Medicine, Houston, Texas (N.J.A.)
}

Received February 18, 2018; accepted March 22, 2018

\begin{abstract}
The sodium/glucose cotransporters (SGLT1 and SGLT2) transport glucose across the intestinal brush border and kidney tubule. Dual SGLT1/2 inhibition could reduce hyperglycemia more than SGLT2-selective inhibition in patients with type 2 diabetes. However, questions remain about altered gastrointestinal (Gl) luminal glucose and tolerability, and this was evaluated in slc5a $1^{-1-}$ mice or with a potent dual inhibitor (compound 8; SGLT1 $K_{\mathrm{i}}=1.5 \pm 0.5 \mathrm{nM} 100$-fold greater potency than phlorizin; SGLT2 $\left.K_{\mathrm{i}}=0.4 \pm 0.2 \mathrm{nM}\right) .{ }^{13} \mathrm{C}_{6}-$ glucose uptake was quantified in slc5a $1^{-1-}$ mice and in isolated rat jejunum. Urinary glucose excretion (UGE), blood glucose (Sprague-Dawley rats), glucagon-like peptide 1 (GLP1), and hemoglobin A1c (HbA1c) levels (Zucker diabetic fatty rats) were measured. Intestinal adaptation and rRNA gene sequencing was analyzed in $\mathrm{C} 57 \mathrm{BI} / 6$ mice. The blood ${ }^{13} \mathrm{C}_{6}$-glucose area under the curve (AUC) was reduced in the absence of
\end{abstract}

SGLT1 by $75 \%(245 \pm 6$ vs. $64 \pm 6 \mathrm{mg} / \mathrm{dl} \cdot \mathrm{h}$ in wild-type vs. slc5a $1^{-1-}$ mice) and compound 8 inhibited its transport up to $50 \%$ in isolated rat jejunum. Compound 8 reduced glucose excursion more than SGLT2-selective inhibition (e.g., AUC = $129 \pm 3$ vs. $249 \pm 5 \mathrm{mg} / \mathrm{dl} \cdot \mathrm{h}$ for $1 \mathrm{mg} / \mathrm{kg}$ compound 8 vs. dapagliflozin) with similar UGE but a lower renal glucose excretion threshold. In Zucker diabetic fatty rats, compound 8 decreased $\mathrm{HbA} 1 \mathrm{c}$ and increased total GLP-1 without changes in jejunum SGLT1 expression, mucosal weight, or villus length. Overall, compound 8 ( $1 \mathrm{mg} / \mathrm{kg}$ for 6 days) did not increase cecal glucose concentrations or bacterial diversity in C57BL/6 mice. In conclusion, potent dual SGLT1/2 inhibition lowers blood glucose by reducing intestinal glucose absorption and the renal glucose threshold but minimally impacts the intestinal mucosa or luminal microbiota in chow-fed rodents.

\section{Introduction}

The sodium/glucose cotransporters SGLT1 and SGLT2 are driven by a sodium gradient created by $\mathrm{Na}^{+} / \mathrm{K}^{+}$ATPase. SGLT1 transports glucose into intestinal enterocytes, whereas SGLT2 (and to a lesser extent SGLT1) reabsorbs glucose in the kidney tubule. SGLT1 is located luminally on

The investigators who are employees of Janssen R\&D LLC and Baylor College of Medicine have no other conflict of interest to disclose.

${ }^{1}$ F.D. and S.A.H. contributed equally to this work as first authors.

https://doi.org/10.1124/jpet.118.248575. the small intestinal brush border in humans (Chen et al., 2010), rats (Balen et al., 2008), and mice (Yoshikawa et al., 2011), with negligible amounts elsewhere in the gastrointestinal (GI) tract. Glutamine is the primary energy source for enterocytes (Frankel et al., 1993) and glucose transporter 2 (GLUT2) transports most glucose basolaterally. Selective SGLT2 inhibitors, marketed for type 2 diabetes mellitus (T2DM), have varying selectivity for SGLT2 relative to SGLT1; for example, canagliflozin has some SGLT1 activity $\left(\mathrm{SGLT} 2 \mathrm{IC}_{50}=4.2 \mathrm{nM}\right.$, SGLT1 $\left.\mathrm{IC}_{50}=663 \mathrm{nM}\right)$ (Mudaliar et al., 2015). Consistent with this in humans,

ABBREVIATIONS: AUC, area under the curve; FITC, fluorescein isothiocyanate; GFR, glomerular filtration rate; GLP-1, glucagon-like peptide 1; GLUT, glucose transporter; GSK-1614235, 3-((3-(4-((5-isopropyl-3-(((2S,3R,4S,5S,6R)-3,4,5-trihydroxy-6-(hydroxymethyl)tetrahydro-2H-pyran2-yl)oxy)-1H-pyrazol-4-yl)methyl)-3-methylphenoxy)propyl)amino)-2,2-dimethylpropanamide; HbA1c, hemoglobin A1c; HFD, high-fat diet; KGA-2727, 3-(3-\{4-[3-( $\beta$-D-glucopyranosyloxy)-5-isopropyl-1H-pyrazol-4-ylmethyl]-3-methylphenoxy\}propylamino)propionamide; KRB, Krebs-Ringer bicarbonate; LC, liquid chromatography; LX2761, N-(1-((2-(dimethylamino)ethyl)amino)-2-methyl-1-oxopropan-2-yl)-4-(4-(2-methyl-5((2S,3R,4R,5S,6R)-3,4,5-trihydroxy-6-(methylthio)tetrahydro-2H-pyran-2-yl)benzyl)phenyl)-butanamide; LX4211, (2S,3R,4R,5S,6R)-2-[4chloro-3-[(4-ethoxyphenyl)methyl]phenyl]-6-methylsulfanyloxane-3,4,5-triol; MS, mass spectrometry; oGTT, oral glucose tolerance test; OTU, operational taxonomic unit; PCR, polymerase chain reaction; $\mathrm{RT}_{\mathrm{G}}$, renal threshold for glucose; SCFA, short-chain fatty acid; SD, Sprague-Dawley; SDI, Shannon diversity index; SGLT, sodium/glucose cotransporter; T2DM, type 2 diabetes mellitus; UGE, urinary glucose excretion; WT, wild type; ZDF, Zucker diabetic fatty. 
transient inhibition of intestinal SGLT1 occurs with $300 \mathrm{mg}$ canagliflozin (Polidori et al., 2013b). In clinical testing, a dual SGLT1/2 inhibitor, sotagliflozin/LX4211 [( $2 S, 3 R, 4 R, 5 S, 6 R)$-2-[4-chloro-3-[(4-ethoxyphenyl)methyl]phenyl]-6-methylsulfanyloxane-3,4,5-triol] $\left(\mathrm{SGLT} 2 \mathrm{IC}_{50}=1.8\right.$ $\mathrm{nM}$, SGLT1 $\mathrm{IC}_{50}=30 \mathrm{nM}$ ), also delayed glucose uptake after an oral glucose or meal test and increased systemic levels of glucagon-like peptide 1 (GLP-1) and peptide YY (Zambrowicz et al., 2012, 2013a). The delay in and augmentation of GLP-1 levels has been noted in diabetic KKAy mice (Powell et al., 2014), healthy humans, and patients with T2DM (Zambrowicz et al., 2013b). The effect of canagliflozin on GLP-1 is enhanced in DPP4-deficient rats (Oguma et al., 2016). The SGLT1-selective inhibitor, GSK-1614235 [3-((3-(4-((5-isopropyl-3-(((2S,3R,4S,5S,6R)-3,4,5-trihydroxy-6-(hydroxymethyl)tetrahydro-2H-pyran-2-yl)oxy)- $1 H$-pyrazol-4-yl)methyl)-3methylphenoxy)propyl)amino)-2,2-dimethylpropanamide], which is an analog of KGA-2727 [3-(3-\{4-[3-( $\beta$-D-glucopyranosyloxy)-5-isopropyl-1H-pyrazol-4-ylmethyl]-3-methylphenoxy\}propylamino)propionamide] with low oral bioavailability, also increases systemic GLP-1 in healthy volunteers (Dobbins et al., 2015). These and other SGLT1 mechanisms in metabolic therapeutics were reviewed recently (Lehmann and Hornby, 2016; Song et al., 2016); however, the extent to which potent inhibition of both SGLT1 and SGLT2 could improve glycemic control has not been explored previously. In this study, we used genetic (slc5a1 ${ }^{-/-}$or slc5a $1^{+/-}$mice) and pharmacological approaches to investigate the effect of acute and chronic dual SGLT1/2 inhibition in mice and rats. Compound 8 was selected from a series of benzocyclobutaneC-glycosides, due to a 100-fold improved potency in SGLT1 inhibition over phlorizin and an approximately equivalent ratio of SGLT1/SGLT2 inhibition (CHO-K1 cells overexpressing SGLT1: compound $8 K_{\mathrm{i}}=1.5 \pm 0.5 \mathrm{nM}$, phlorizin $K_{\mathrm{i}}=155.8 \mathrm{nM}$ in the same assay; CHO-K1 overexpressing SGLT2: $K_{\mathrm{i}}=0.4 \pm 0.2 \mathrm{nM}$ ). Compound 8 had a terminal half-life of approximately 2.5 hours in rodents (Kuo et al., 2018), which is suitable for assessing glycemic control in vivo.

Despite the promising therapeutic benefits of SGLT1 inhibition, there has been a modest increase in subjects reporting GI adverse effects of mild or moderate intensity (Dobbins et al., 2015). Functional mutations within the Slc5a1 gene in humans are associated with severe diarrhea (Vallaeys et al., 2013; Pode-Shakked et al., 2014). Gut-restricted SGLT1selective inhibitors, such as LX2761 [N-(1-((2-(dimethylamino) ethyl)amino)-2-methyl-1-oxopropan-2-yl)-4-(4-(2-methyl-5((2S,3R,4R,5S,6R)-3,4,5-trihydroxy-6-(methylthio)tetrahydro$2 H$-pyran-2-yl)benzyl)phenyl)-butanamide], can be associated with diarrhea in rodents (Fushimi et al., 2013; Powell et al., 2017). An SLGT1-selective inhibitor, mizagliflozin, administered before each meal to healthy volunteers increased stool frequency (Inoue et al., 2017), although sotagliflozin did not promote defecation (Lapuerta et al., 2015). In rats, high doses of canagliflozin increased colonic glucose concentration and decreased $\mathrm{pH}$, presumably due to microbial metabolism of glucose to shortchain fatty acids (SCFAs) (De Jonghe et al., 2014). Reducing nutrient absorption by bariatric surgery in obese rats resulted in mucosal adaptation and changes in SGLT1 expression (Cavin et al., 2016). Therefore, the small intestine was evaluated for mucosal adaptation and SGLT1/GLUT2 expression after chronic SGLT1/2 inhibition in rats and for altered luminal glucose concentrations and bacterial diversity and abundance in mice.

\section{Materials and Methods}

Animals. All reported studies on rats and mice were carried out in accordance with the recommendations in the National Institutes of Health Guide for the Care and Use of Laboratory Animals. The protocols for all studies performed at Janssen Pharmaceuticals were approved by the Johnson \& Johnson Animal Care and Use Committee.

Age-matched C57Bl/6 wild-type (WT) mice and fully back-crossed mice lacking one allele (heterozygous) or both alleles (homozygous null) of Sglt1 (slc5a1) were obtained from Taconic Biosciences (Hudson, NY) at age 20-22 weeks. Mice were singly housed and fed a 60\% high-fat, low-carbohydrate diet ad libitum (D12492; Research Diets, New Brunswick, NJ). Male Sprague-Dawley (SD) rats were obtained from Charles River Laboratories International (Wilmington, MA) at age 7 to 8 weeks and fed a chow diet (Chow Diet 5K75; Research Diets). Male Zucker diabetic fatty (ZDF) rats were obtained from Charles River Laboratories at age 12 to 13 weeks and fed a chow diet (Formulab Diet 5008; Research Diets). All mice and rats (two per cage) were maintained in a temperature-controlled environment with a 12-hour/12-hour light/dark cycle (lights off, 1800; lights on, 0600) and had free access to water and food.

Blood glucose levels were measured using One-Touch glucometers (LifeScan Inc., Chesterbrook, PA) via a tail snip. Urine glucose levels were determined by the Olympus system with Genzyme diagnostics assay reagent (Sekisui Diagnostics, Lexington, MA). ${ }^{13} \mathrm{C}_{6}$-glucose levels were determined using liquid chromatography (LC)-tandem mass spectrometry (MS) combined with dried blood spot sampling technology (Lang et al., 2014). Blood hemoglobin A1c (HbA1c) levels were tested using a Siemens DCA Vantage analyzer (Siemens, Munich, Germany). Plasma insulin and total GLP-1 concentrations were detected using electrochemiluminescent immunoassays (Meso Scale Discovery, Rockville, MD).

SGLT1 Genotype Mouse Studies. Oral glucose tolerance tests (oGTTs) were performed on conscious, unrestrained mice. Blood glucose levels of mice fasted overnight were measured, and the mice then received $2 \mathrm{~g} / \mathrm{kg}{ }^{13} \mathrm{C}_{6}$-glucose (Cambridge Isotope Laboratories, Andover, MA) and glucose (1:1 ratio) by oral gavage. Blood levels of glucose and ${ }^{13} \mathrm{C}_{6}$-glucose were measured at $0,10,30,60,120,180$, and 240 minutes after mice were given the ${ }^{13} \mathrm{C}_{6}$-glucose challenge. Insulin tolerance tests were performed using human insulin (Humulin R; Eli Lilly, Indianapolis, IN). After 4-hour food removal, body weight and baseline blood glucose levels were measured and then insulin $(0.75$ $\mathrm{U} / \mathrm{kg}$ s.c.) was injected and blood glucose levels were measured at 0,15 , 30,60 , and 120 minutes.

RNA was purified from mouse mucosa tissue utilizing the RNeasy Mini Kit (Qiagen, Hilden, Germany). In brief, tissues were homogenized in $500 \mu \mathrm{l}$ lysis buffer with 5-mM-diameter sterile steel ball bearings in a tissue lyser (TissueLyser II; Qiagen) for 4 minutes at $30 \mathrm{~Hz}$. Homogenates were spun down at 12,000 rpm for 2 minutes at $4^{\circ} \mathrm{C}$, and RNA was purified from supernatants according to the RNeasy protocol, utilizing the on-column DNase digestion additional quality control procedure (Qiagen). cDNA was generated by reverse transcription using the HighCapacity cDNA Reverse Transcription Kit (Thermo Fisher, Waltham, MA) according to the manufacturer's protocols. qRT-polymerase chain reaction (PCR) was conducted using the viia-7 real-time PCR machine and SYBR Green Master Mix (Thermo Fisher). Primer sequences were as follows: SLC5A1 (forward, 5' -CCCTGTGGTACTGGTGTACG-3'; reverse, $5^{\prime}$-ATCATTCCTGGCATCACCAT-3'), SLC2A2 (forward, 5' CTGTCTGTGTCCAGCTTTGC-3'; reverse, 5' -ATCAAGAGGGCTCCAGTCAA-3'), SLC2A1 (forward, 5'-GTATCCTGTTGCCCTTCTGC-3'; reverse, $5^{\prime}$-ATCTGCCGACCCTCTTCTTT-3'), and glyceraldehyde-3phosphate dehydrogenase (forward, 5'-GACTGGATAAGCAGGGCGG3'; reverse, 5'-TACGGCCAAATCCGTTCACA-3'). The run method for qRT-PCR analysis consisted of the following: 1) hold state: $50^{\circ} \mathrm{C}$ for 2 minutes, $95^{\circ} \mathrm{C}$ for 3 minutes; 2) PCR stage: $95^{\circ} \mathrm{C}$ for 15 seconds (melt stage), $60^{\circ} \mathrm{C}$ for 30 seconds (annealing and amplification) $\times$ 40 cycles; and 3) melt curve for primer quality control. Results are represented by the fold change (where fold change equals $2^{-\Delta \Delta \mathrm{CT}}$ ). 
Glyceraldehyde-3-phosphate dehydrogenase served as the housekeeping control gene.

SD Rat Studies. For Ussing-type flux chamber experiments with isolated jejunum, fed rats were euthanized using $\mathrm{CO}_{2}$ and the middle one-third of the small intestine (jejunum) was resected and washed with ice-cold Krebs-Ringer bicarbonate (KRB) buffer aerated with 95\% $\mathrm{O}_{2}-5 \% \mathrm{CO}_{2}$. A 2-cm segment of rat jejunum was cut and placed onto a knitting needle (no. 8). Excess fat was removed and the outer intestinal muscle layer was scored using Dumont forceps. Cotton swabs were used to push away the serosal muscle layer so that it rolled over the mucosal layer, allowing it to then be removed. The mucosal layer was gently pulled off the knitting needle into ice-cold KRB buffer and opened longitudinally and then positioned in a cassette cartridge, which had an exposed a surface area of $0.1 \mathrm{~cm}^{2}$ of the intestine. The cassette was placed into the chambers dividing the mucosal/apical sides from the serosal/basolateral sides. The apical chamber contained a modified KRB buffer recipe, which included $12.8 \mathrm{mM} \mathrm{C-13-D-glucose}$ rather than the typical $12.8 \mathrm{mM}$ D-glucose. Basolateral side buffer samples' C-13-D-glucose concentrations were quantified using Nimbus LC-MS against internal standards [chromatographic description: LC-20AD pumps with an SIL-20AC autosampler (Shimadzu, Kyoto, Japan); MS description: MDS Sciex API 5000 with an APCI source (Applied Biosystems, Foster City, CA)].

Fluorescein isothiocyanate (FITC)-dextran (Sigma, St. Louis, MO) was used to determine intestinal tissue integrity. FITC-dextran was added to the serosal/basolateral KRB buffer at a final concentration of $20 \mu \mathrm{g} / \mathrm{ml}$. Relative fluorescence units were measured on a Safire ${ }^{2}$ plate reader with an excitation wavelength of $492 \mathrm{~nm}$ (slit width $=10.0 \mathrm{~nm}$ ) and an emission wavelength of $525 \mathrm{~nm}$ (slit width $=10.0 \mathrm{~nm}$ ). Compound 8 in vitro potency $(0.001-10 \mu \mathrm{M})$ was previously evaluated using methyl-glucopyranoside (Sigma) in CHO-K1 cells stably expressing SGLT1 or SGLT2, as reported (Kuo et al., 2018).

For in vivo studies, rats were fasted overnight and were treated orally or subcutaneously with vehicle $(0.5 \%$ HPMC) or compounds 30 minutes prior to oGTTs at 30 minutes and 6 hours after treatment. Blood glucose levels were measured at $0,30,60$, and 120 minutes after glucose challenge. Rats were housed in metabolic cages for urine collection to detect urinary glucose excretion (UGE) up to 24 hours after oGTTs.

The renal threshold for glucose $\left(\mathrm{RT}_{\mathrm{G}}\right)$ was calculated as follows. To account for between-group differences in blood glucose levels when interpreting UGE data, values of $\mathrm{RT}_{\mathrm{G}}$ were calculated to assess inhibition of renal glucose reabsorption. Previously obtained glomerular filtration rates (GFRs) based on creatine clearance were used to calculate $\mathrm{RT}_{\mathrm{G}}$ over each urine collection interval, as previously described (Sha et al., 2011; Polidori et al., 2013a). In short, an idealized threshold relationship was used to describe the relationship between blood glucose and UGE (eq. 1):

$$
\text { Rate of } \operatorname{UGE}(\mathrm{mg} / \mathrm{min})= \begin{cases}\mathrm{GFR} \times\left(\mathrm{BG}-\mathrm{RT}_{\mathrm{G}}\right) & \text { if } \mathrm{BG}>\mathrm{RT}_{\mathrm{G}} \\ 0 & \text { if } \mathrm{BG} \leq \mathrm{RT}_{\mathrm{G}}\end{cases}
$$

This equation, along with measured values of UGE and blood glucose (BG) over each urine collection interval, and estimated GFR values were used to calculate $\mathrm{RT}_{\mathrm{G}}$. Because even untreated, normoglycemic animals tend to have trace amounts of UGE, values of $\mathrm{RT}_{\mathrm{G}}$ were only calculated when UGE was at least $20 \mathrm{mg}$. The 24 -hour mean $\mathrm{RT}_{\mathrm{G}}$ values were calculated as the weighted mean of the values obtained over each urine collection interval. In several cases, SD rats treated with compounds had a measured UGE greater than the calculated filtered glucose load (which is GFR $\times$ BG). In these cases, $\mathrm{RT}_{\mathrm{G}}$ was set to 0 , which assumes no renal glucose reabsorption occurred (consistent with the measured UGE being even greater than the filtered glucose load). In vehicle-treated SD rats (and some SD rats treated with low doses of SGLT inhibitors), virtually no UGE was observed. In these rats, a value of $225 \mathrm{mg} / \mathrm{dl}$ was imputed based on (unpublished) data obtained with glucose infusion studies.
For subcutaneous administration of compound 8 , the vehicle was $10 \%$ PEG-400/10\% Solutol $(n=4)$ and rats were euthanized at 1 and 6 hours. To calculate the tissue/plasma ratio, routine LC-MS was used to detect compound 8 in the tissue (kidney and intestinal luminal contents, mucosal scraping, and serosa) and plasma.

ZDF Rat Studies. Male ZDF rats (aged 12-14 weeks) had blood glucose levels that were $\geq 350 \mathrm{mg} / \mathrm{dl}$ at the start of approximately 3 weeks of treatment while they were maintained on a reverse light cycle. On day 1, ZDF rats were allocated to different treatment groups based on their body weights and baseline fed blood glucose levels. Then animals were orally dosed with vehicle or compound 8 every day for 18 days. Fed blood glucose, body weights, and food weights were measured every 4 days. On day 15 blood HbA1c levels were measured On day 16 , an oGTT with a $0.5 \mathrm{~g} / \mathrm{kg}$ glucose challenge and total GLP-1 levels were measured at 0 and 60 minutes of oGTT. On day 19, 24 hours after the final $1 \mathrm{mg} / \mathrm{kg}$ dose, the rats were anesthetized and intestine and kidney tissue $(n=4)$ were collected. The plasma tissue levels of compound 8 were detected by LC-MS in the intestinal mucosa, serosa/muscularis, and kidneys.

For immunohistochemistry, 1-cm segments of jejunum were opened longitudinally along the mesenteric border, placed flat on biopsy sponges, and fixed in $10 \% \mathrm{NBF}$ for 24 hours and then transferred to $70 \%$ alcohol for later paraffin embedding for histology. After routine dehydration and paraffin embedding, seven coded blocks of tissues, each with three to four tissues totaling 19 jejunum tissues, were processed for immunohistochemistry. Routine chromagen staining immunohistochemistry methods were modified to accommodate the suggested EDTA ( $\mathrm{pH}$ 9.0) retrieving pretreatment solution. The SGLT1 polyclonal antibody (Abcam, Cambridge, MA), negative control (no primary antibody), and positive control antibody (von Willebrand factor) were used for each tissue.

SGLT1 Inhibition of Large Intestine Glucose in Mice and 16s rRNA Analysis. Male C57Bl/6 mice were dosed with $1 \mathrm{mg} / \mathrm{kg}$ compound 8 for 5 days in the late afternoon prior to the dark cycle and were fed regular chow or high-sucrose (35\%) diets. Control mice were fed regular chow and dosed with vehicle. Body weight, fasted (4-hour) blood glucose, fed blood glucose, gross cecal weight, and cecal glucose content were measured. At harvest on day 6 , the cecum was ligated at the ileocecal junction and the entire weight including contents was recorded for calculation of the percentage of cecal weight to body weight. Cecal contents were diluted $1: 1$ with $0.9 \% \mathrm{NaCl}$ and placed on wet ice and vortexed $1500 \mathrm{~g}$ at $4^{\circ} \mathrm{C}$ for 10 minutes, and the supernatant sample was taken for glucose analysis by an Autokit Glucose kit (Wako, Osaka, Japan) using a SpectraMax Plus384 plate reader (Molecular Devices). In addition to comparing cecal bacterial composition at harvest, fecal bacterial diversity was assessed in fecal pellets collected at days 0,2 , and 5 to understand dynamic changes. All cecal and fecal samples (72 samples total) were shipped to Diversigen Inc. (Houston, TX) for 16S rRNA gene sequencing, which included extraction, PCR amplification, sequencing (MiSeq; $2 \times 250 \mathrm{bp}$; Illumina, San Diego, CA), and analysis (Caporaso et al., 2011, 2012). Mouse cecal contents and fecal pellet sequencing identified 235 operational taxonomic units (OTUs) with a cutoff of $4074 \mathrm{reads} / \mathrm{sample}$. All samples were included in the analysis of bacterial richness, diversity, and taxonomic relative abundance.

All statistical analysis was performed by parametric $t$ tests or analysis of variance with Bonferroni, Tukey, or Dunnett multiple comparisons post hoc tests (GraphPad Prism, version 7.0; Graph Pad Software Inc., La Jolla, CA). Statistical significance was defined as $P<0.05$. A Kruskal-Wallis nonparametric analysis of variance was used to analyze terminal insulin levels in slc5a $1^{+/-}$mice, due to high variability without normal distribution.

\section{Results Was Done}

Glucose homeostasis was assessed in genetically modified slc5a1 homozygous null (slc5a $1^{-/-}$) or heterozygous $\left(\mathrm{slc}_{\mathrm{a}} \mathrm{a}^{+/-}\right.$) mice and compared with age-matched WT c57 control mice. 
Ileal and jejunal mucosa isolated from WT mice had robust expression of slc5a1 mRNA that was essentially absent in slc5a1 $1^{-/-}$mice and intermediate between those in slc5a1 ${ }^{+/-}$ mice (Fig. 1A) that had been fed a high-fat diet (HFD). GLUT2 mRNA expression was similar in all groups, consistent with normal facilitated transport of absorbed glucose (data not shown). An oGTT $\left({ }^{13} \mathrm{C}\right.$-glucose/glucose $\left.1: 1\right)$ resulted in a glucose excursion as measured by glucometer that was markedly lower in slc5a1 $1^{-1-}$ mice compared with WT and heterozygous mice (Fig. 1B). Detection of ${ }^{13} \mathrm{C}$-glucose for absorbed glucose (Fig. 1C) illustrated that the peak levels were reduced to $<25 \%$ of slc5a1 $1^{+/-}$and WT peak levels in slc5a1 ${ }^{-/-}$ mice. This difference was reflected in the area under the curve (AUC) for ${ }^{13} \mathrm{C}_{6}$-glucose, which was approximately $25 \%$ of $\mathrm{WT}$ in slc5a1 $1^{-1-}$ mice (Fig. 1D). Consistent with these glucose levels, terminal insulin levels in slc5a1 ${ }^{-1-}$ mice were low compared with WT and slc5a1 ${ }^{+/-}$mice (Fig. 1E). An increase in endogenous SGLT1 expression has been reported in obesity;
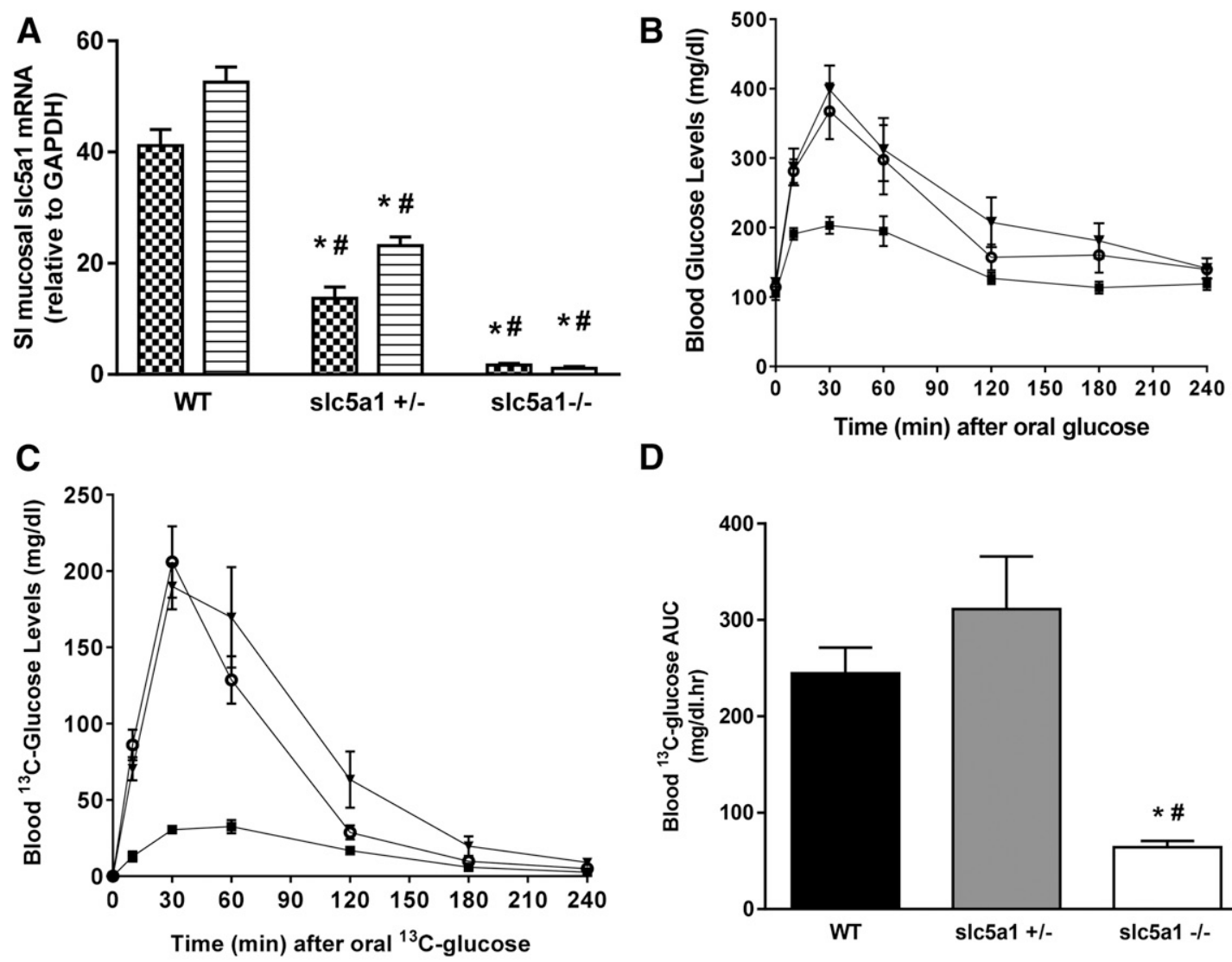

D
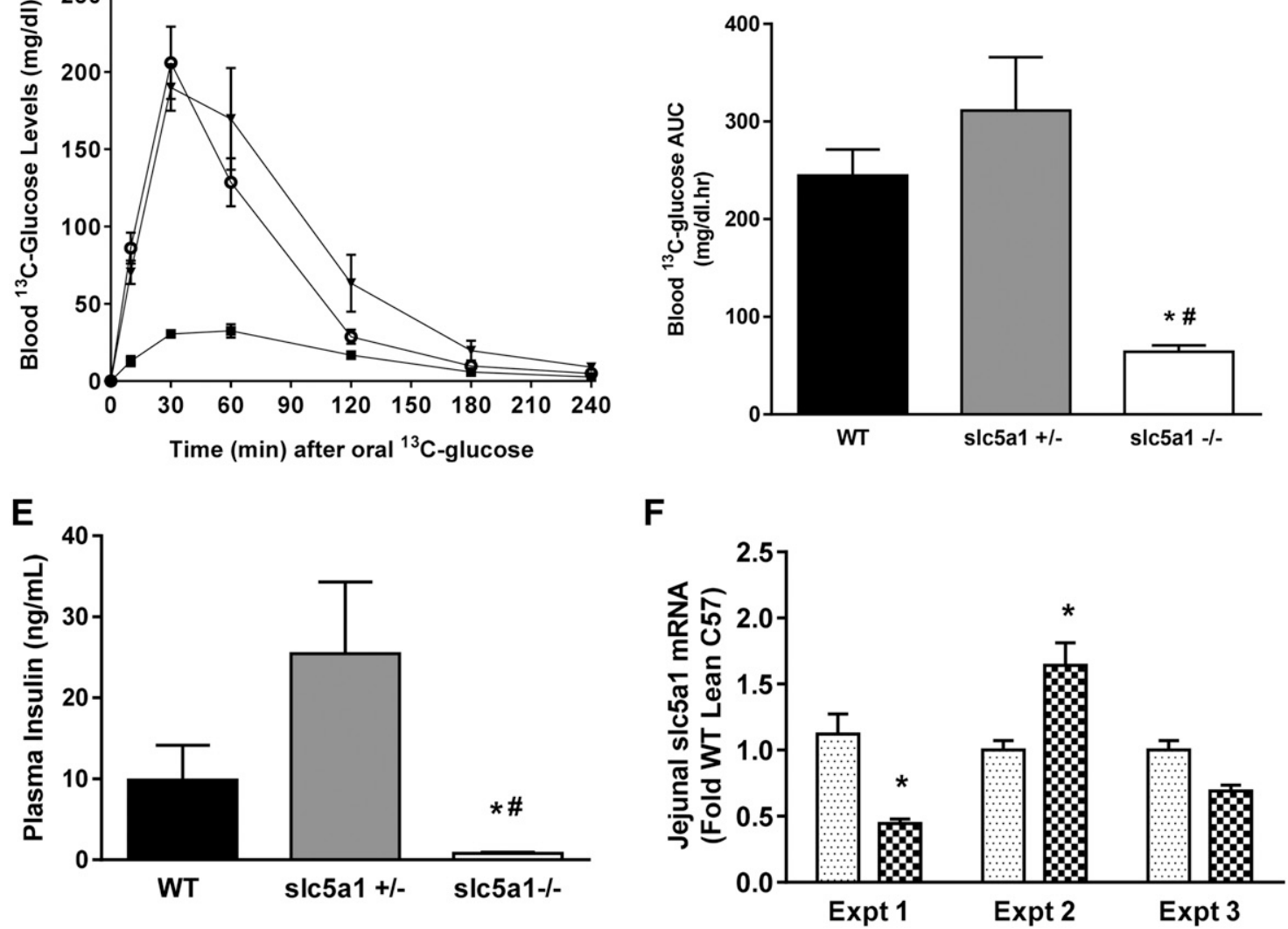

Fig. 1. Glucose homeostasis and slc5a1 mRNA in slc5a1 $1^{-/-}$, slc5a1 $1^{+/-}$, and WT mice. (A) slc5a1 mRNA levels in jejunal (cross-hatched bars) and ileal (horizontal bars) mucosa were low in homozygous slc5a1 ${ }^{-/-}$mice and intermediate in slc5a1 $1^{+1}$ mice compared with WT $(n=7$ to $8 /$ group). $* P<0.05$ (compared with WT); ${ }^{\#} P<0.05$ (compared with slc5a $1^{+/-}$). (B) Glucose excursion after an oGTT $\left(50 \%{ }^{13} \mathrm{C}_{6}\right.$-glucose and $50 \%$ glucose) in slc5a $1^{-/-}$mice (squares) was markedly lower than in WT (circles) and heterozygous mice (triangles). (C) Blood ${ }^{13} \mathrm{C}_{6}$-glucose excursions were similar to glucose, but with a better separation among the three animal models. (D) Blood glucose AUC in the same mice. ${ }^{*} P<0.05$ (compared with WT; black bar); ${ }^{\#} P<0.05$ (slc5 ${ }^{+/}$ compared with slc5a $1^{-1-}$ mice; gray bar and white bar, respectively). (E) Terminal plasma insulin levels in the same mice, showing that insulin was lower in slc5a1 ${ }^{-/-}$mice than both WT $(* P<0.05)$ and slc5a $1^{+/-}$mice ( ${ }^{\#} P<0.05$ by Kruskal-Wallis nonparametric analysis of variance due to high variability. (F) Jejunal mucosa slc5a1 expression in HFD-fed DIO mice (cross-hatched bars) was not consistently changed relative to lean chow-fed WT mice (stippled bars) in three separate experiments ( $n=7$ to 8/group). GAPDH, glyceraldehyde-3-phosphate dehydrogenase; DIO, diet-induced obese. 

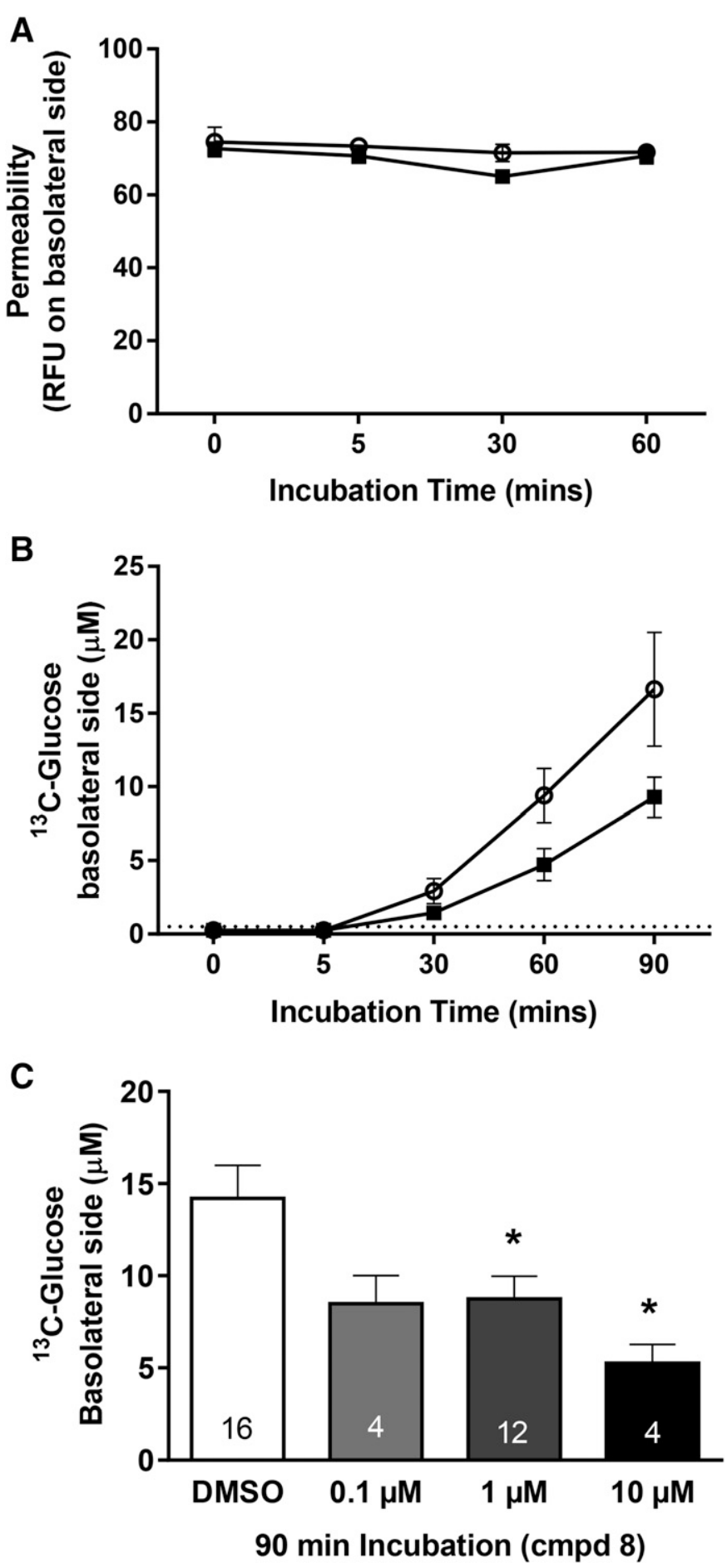

Fig. 2. Muscle-stripped rat jejunum mounted in Ussing-type flux chambers in the presence and absence of SGLT1 inhibitor compound $8(1 \mu \mathrm{M}$ in DMSO). (A) Mucosal tissue integrity was established in initial experiments by FITC-dextran detection $<100$ RFU on the basolateral side after 4-kDa FITC-conjugated dextran was added to the apical side in the presence of vehicle DMSO (circles) and compound 8 (squares). (B) Time course of ${ }^{13} \mathrm{C}$-glucose detection on the basolateral side in the presence of vehicle alone or with compound $(1 \mu \mathrm{M})(n=3-9$ per time point). (C) Concentration-related inhibition of ${ }^{13} \mathrm{C}$-glucose uptake at the 90-minute time point by compound $8(0.1-10 \mu \mathrm{M}$, gray-black bars) and vehicle (white bar). Numbers in the bars indicate replicates. ${ }^{*} P<0.05$ (by one-way analysis of variance and the Dunnett multiple comparison test). The blood ${ }^{13} \mathrm{C}$ glucose detection LLOQ was $0.5 \mu \mathrm{M}$ (shown as a dashed line) and any samples below this were plotted as one-half of the LLOQ. DMSO, dimethylsulfoxide; LLOQ, lower limit of quantification; RFU, relative fluorescence unit. therefore, we assessed jejunum slc5a1 mRNA expression in diet-induced obese $\mathrm{C} 57 \mathrm{Bl} / 6 \mathrm{WT}$ mice. However, different results were obtained in three repeat experiments of no change, upregulation, and downregulation in slc5a1 mRNA expression in diet-induced obese mice relative to lean controls (Fig. 1F)

Endogenous SGLT1 engagement was assessed by ${ }^{13} \mathrm{C}_{6^{-}}$ glucose transit across isolated muscle-stripped jejunum in Ussing-type flux chambers using dual SGLT1/2 inhibitor compound 8 (Kuo et al., 2018). Tissue integrity of the jejunal mucosa, as measured by FITC-dextran detection on the basolateral side after apical administration, was acceptable and stable over time in the presence of dimethylsulfoxide with or without compound 8 (Fig. 2A). Thus, in subsequent experiments, FITC-dextran detection of $>150$ relative fluorescence units after 90-minute incubation was discarded from ${ }^{13} \mathrm{C}$ glucose analysis. Compound $8(1 \mu \mathrm{M})$ reduced the uptake of apically applied ${ }^{13} \mathrm{C}$-glucose over time compared with dimethylsulfoxide vehicle alone (Fig. 2B). At 90 minutes, compound 8 (1 and $10 \mu \mathrm{M})$ inhibited ${ }^{13} \mathrm{C}_{6}$-glucose detection on the basolateral side relative to vehicle but did not completely abolish it even at $10 \mu \mathrm{M}$ (Fig. 2C).

In SD rats, compound 8 (0.1-10 $\mathrm{mg} / \mathrm{kg}$ p.o.) given 30 minutes prior to an oGTT reduced glucose excursion (Fig. 3A), glucose AUC (Fig. 3C), and UGE (Fig. 3E). Blood glucose levels were significantly reduced by all doses of compound 8 and the three highest doses of a potent SGLT2-selective inhibitor, dapagliflozin (Fig. 3, B and D). Furthermore, UGE at 0-24 hours was comparable for both compound 8 (Fig. 3E) and dapagliflozin (Fig. 3F), although blood glucose levels were lower with compound 8 than with dapagliflozin. As a result, the mean $\mathrm{RT}_{\mathrm{G}}$ values were lower for compound 8 (Fig. 3G) than for dapagliflozin (Fig. 3H); at the highest doses of compound 8 , complete inhibition of renal glucose reabsorption was achieved, as evidenced by $\mathrm{RT}_{\mathrm{G}}$ values of approximately 0 .

Subcutaneous (instead of oral) administration of compound $8(0.3$ and $10.0 \mathrm{mg} / \mathrm{kg})$ increased UGE $0-4$ hours after dosing 30 minutes prior to an oGTT (Fig. 4A), as would be expected due to renal SGLT inhibition. Subcutaneous administration of compound 8 also prevented the postprandial blood glucose excursion (Fig. 4, B and $\mathrm{C}$ ) in a similar fashion to oral administration. At termination, 6 hours after subcutaneous dosing, plasma levels were low for both doses of compound $8(0.06 \pm 0.03$ and $0.07 \pm 0.00 \mu \mathrm{M})$. The tissue/plasma ratio was elevated in the kidney and in the small intestinal, but not colonic, mucosa (Fig. 4D). The ratio of compound 8 levels in small intestinal luminal content to plasma was low, but the colonic luminal contents had an elevated ratio, which could arise from sloughed off intestinal enterocytes containing compound 8. Combined, these data suggest that compound 8 rapidly reaches SGLT1 in the small intestinal brush border after parenteral administration.

In $\mathrm{ZDF}$ rats, compound $8(0.3$ and $1.0 \mathrm{mg} / \mathrm{kg}$ p.o. for 15 days) reduced fed blood glucose (Fig. 5A), the AUC of an oGTT (Fig. 5B), and circulating HbA1c levels (Fig. 5C) compared with vehicle. At the same time, the higher dose increased total circulating GLP-1 (Fig. 5D) and body weight gain (Fig. 5E), whereas food intake remained the same over the entire study (data not shown). At 3 weeks, animals euthanized at 24 hours after the last dose of compound 8 had plasma levels below the level of quantification; however, detectable levels were noted in the small intestine and 

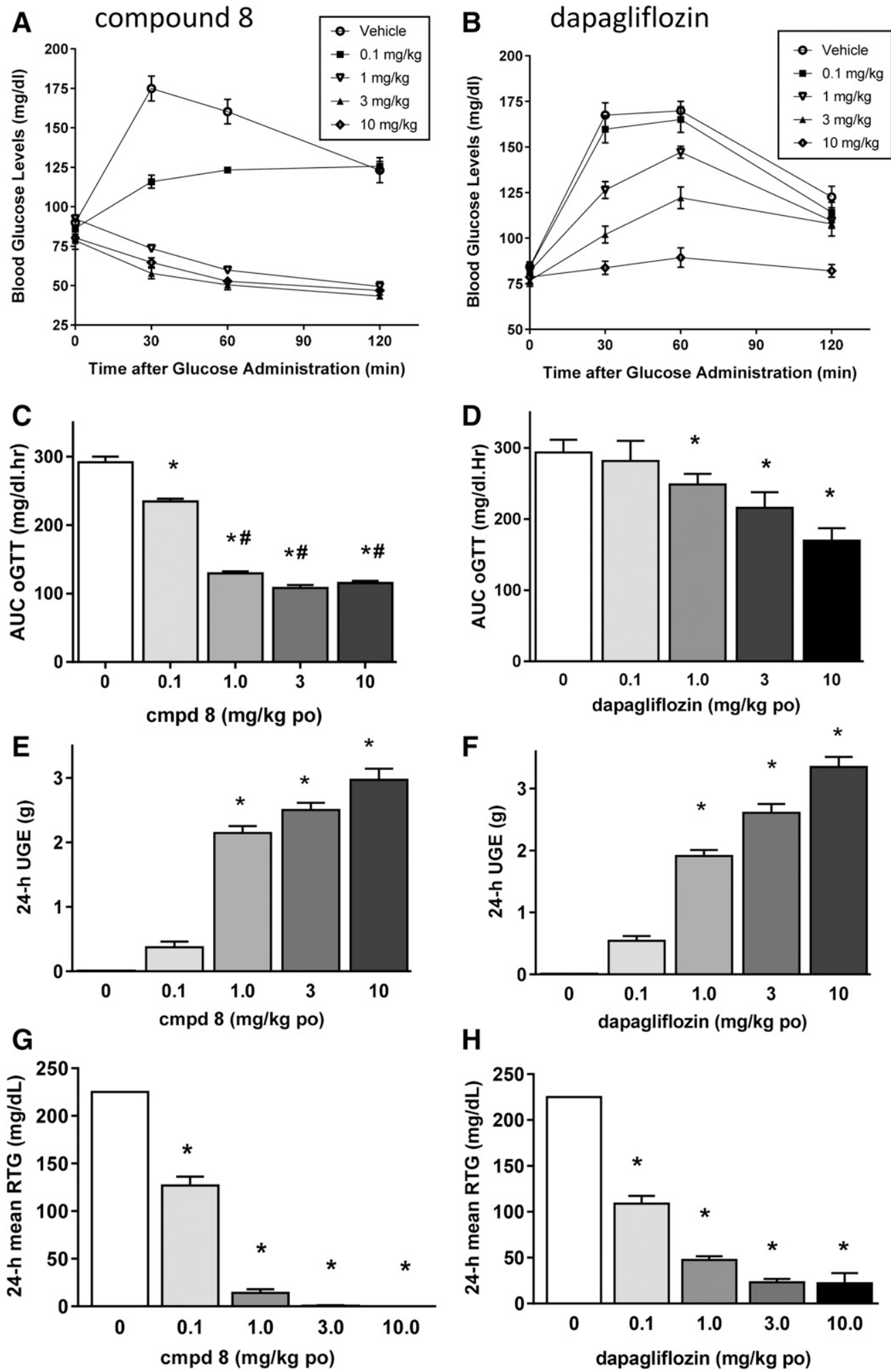

Fig. 3. (A-H) SGLT1/2 dual inhibition (compound 8; A, C, E, and G) and SGLT2-selective inhibition (dapagliflozin; B, D, F, and H) dosed orally in SD rats reduced glucose excursion after oGTT and increased UGE. (A and B) Blood glucose excursions (0.1-10 mg/kg compound 8 or dapagliflozin given 30 minutes prior) were reduced in a dose-related manner compared with vehicle. (C and D) Glucose AUC during the oGTT was significantly reduced by all doses of compound 8 and the three highest doses of dapagliflozin. (E and F) Comparable increase in UGE over 0-24 hours for both compound 8 and dapagliflozin at $1-10 \mathrm{mg} / \mathrm{kg}$. (G and $\mathrm{H}$ ) During the same time, mean $\mathrm{RT}_{\mathrm{G}}$ values were lower for compound 8 than dapagliflozin and complete inhibition of renal glucose was achieved at the highest doses of compound $8 . *=P<0.05$ by analysis of variance or the Tukey or Dunnett multiple comparisons test.

kidney at both the 0.3 (data not shown) and $1.0 \mathrm{mg} / \mathrm{kg}$ doses (Fig. $5 \mathrm{~F}$ ). In the jejunum mucosa of these rats, there was no difference in the level of expression of mRNA encoding
SGLT1, GLUT1, or GLUT2 mRNA after dosing with compound 8 compared with vehicle (Fig. 5G). Consistent with this, jejunum brush border membrane SGLT1 
A

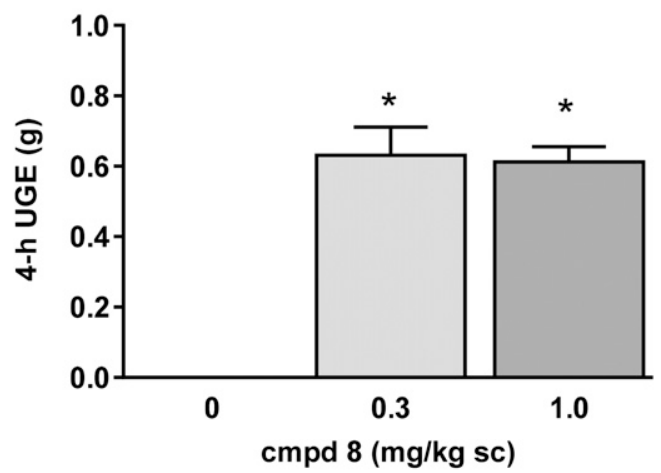

C

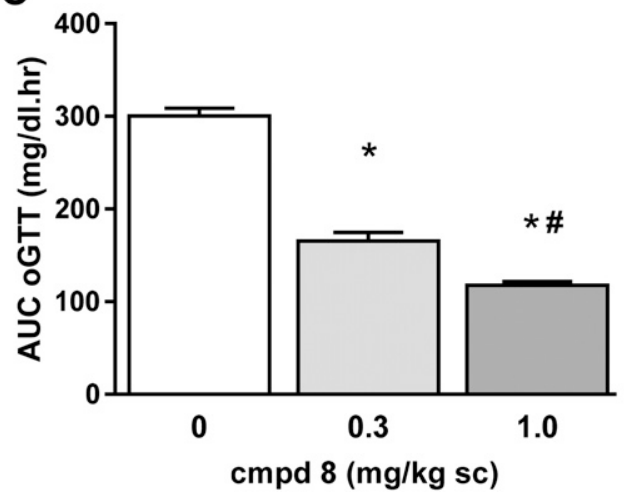

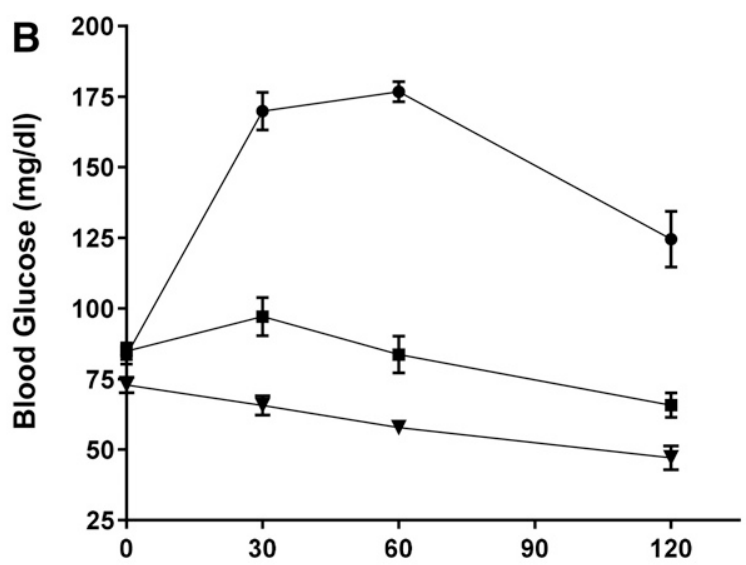

Time after Glucose Administration (min)

D

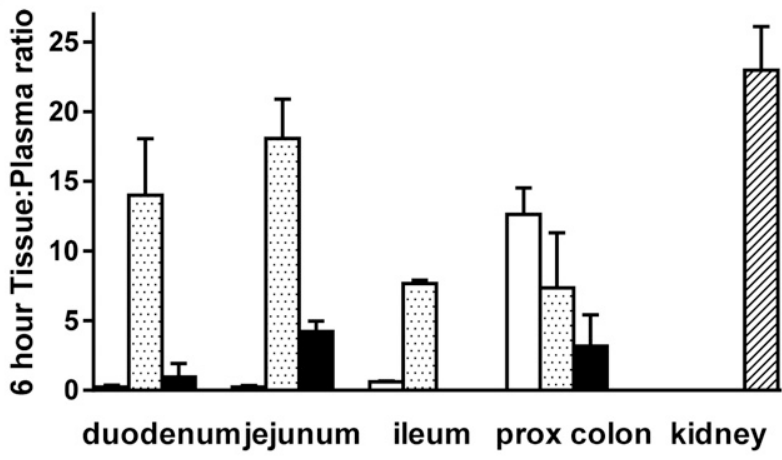

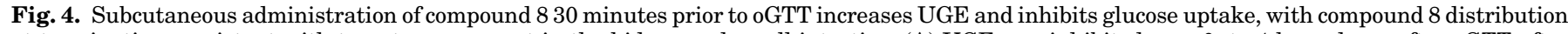

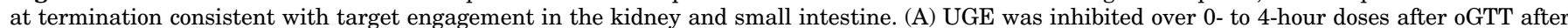

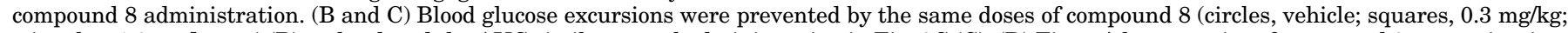

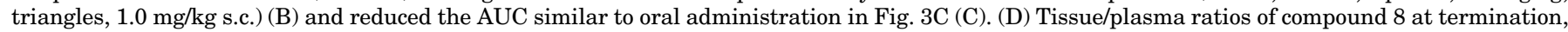

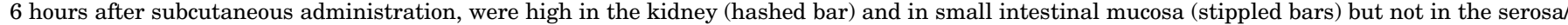

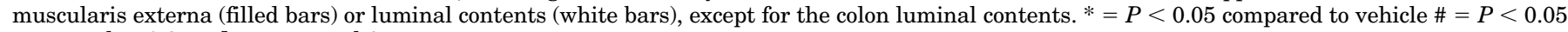
compared to $0.3 \mathrm{mg} / \mathrm{kg}$ compound 8 .

immunostaining was qualitatively similar in all groups ( $n=4$ /group). Representative digital images illustrate that approximately $90 \%$ of the brush border had moderate to strong SGLT1 expression across all groups (Fig. 5H). There was no apparent small intestinal mucosal adaptation, since the gross jejunum mucosal weight of a 2 -cm segment was similar in all groups $(0.19 \pm 0.02,0.19 \pm 0.01$, and $0.23 \pm$ $0.02 \mathrm{~g}$ for vehicle, $0.3 \mathrm{mg} / \mathrm{kg}$, and $1.0 \mathrm{mg} / \mathrm{kg}$, respectively; $n=4-8$ /group). Morphometric analysis ( $n=4$ /group) confirmed that there was no difference in the villus/crypt ratio or villus length $(671 \pm 36,496 \pm 20$, and $651 \pm 18 \mu \mathrm{m}$ for vehicle, $0.3 \mathrm{mg} / \mathrm{kg}$, and $1.0 \mathrm{mg} / \mathrm{kg}$, respectively). There was no difference in cecal content glucose concentration $(23.3 \pm 7.5$, $29.2 \pm 5.1$, and $26.8 \pm 3.6 \mathrm{mg} / \mathrm{dl}$ for vehicle, $0.3 \mathrm{mg} / \mathrm{kg}$, and $1.0 \mathrm{mg} / \mathrm{kg}$, respectively; $n=8 /$ group $)$ or $\mathrm{pH}(6.3 \pm 0.1,6.3 \pm$ 0.1 , and $6.4 \pm 0.1$ for vehicle, $0.3 \mathrm{mg} / \mathrm{kg}$, and $1.0 \mathrm{mg} / \mathrm{kg}$, respectively; $n=8$ /group).

Mice were fed chow or a high-sucrose diet combined with compound 8 ( $1 \mathrm{mg} / \mathrm{kg}$ p.o. for 6 days). At termination, gross cecal weight was decreased in mice fed a high-sucrose diet and treated with compound 8 (Fig. 6A) but overall the cecal glucose concentration was similar (Fig. 6B). There was no difference in body weight or fed blood glucose levels (data not shown) and fecal pellet consistency and wet weight were similar in all groups (data not shown). Although cecal glucose was not different, there was an increase in variability in chow-fed mice treated with compound 8. However, when we compared cecal bacterial diversity, as measured by the number of observed OTUs, there was no correlation with cecal glucose concentration in the same mice as shown by $16 \mathrm{~S}$ rRNA gene sequencing (Fig. 6C). At termination, the relative abundance of certain phyla represented in cecal contents only differed in mice fed a high-sucrose diet with compound 8. In these mice, higher Bacteroidetes and lower Firmicutes were represented compared with mice fed chow with compound 8 or mice fed chow plus vehicle (Fig. 6D). Cecal and fecal sample microbial diversity, collected at the termination of the study, was highly correlated, as measured by the Shannon diversity index (SDI; Fig. 6E). Longitudinal analysis alone of fecal SDI changed on day 2 after mice were switched to a high-sucrose diet with compound 8 and this diversity was maintained to day 6 (Fig. $6 \mathrm{~F}$ ), whereas the addition of compound 8 or vehicle in chow-fed mice was not different throughout the study. The relative abundance of bacterial orders, as well as bacteria of interest in metabolic disease (e.g., Akkermansia spp.), was not altered by compound 8 (data not shown). 

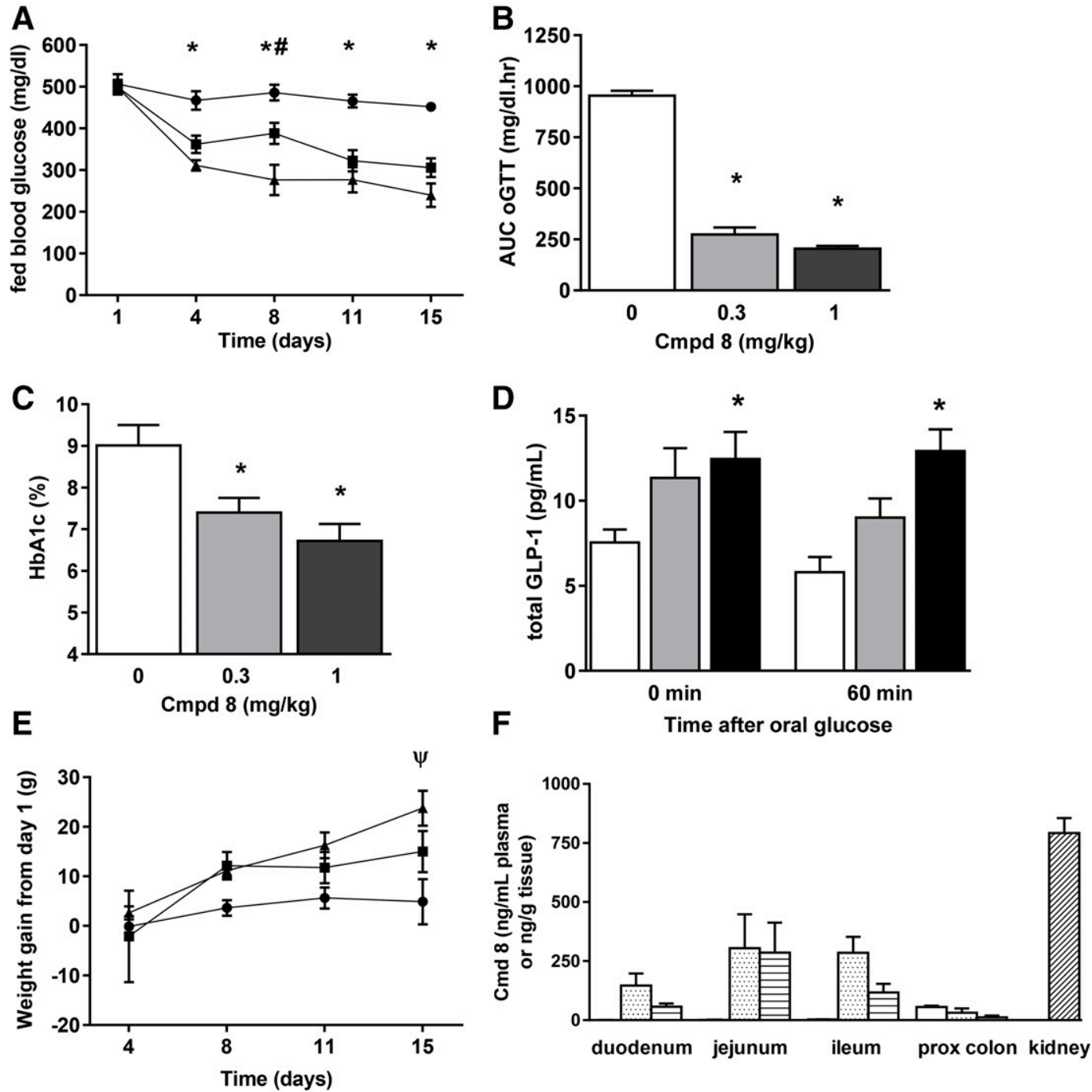

G

H
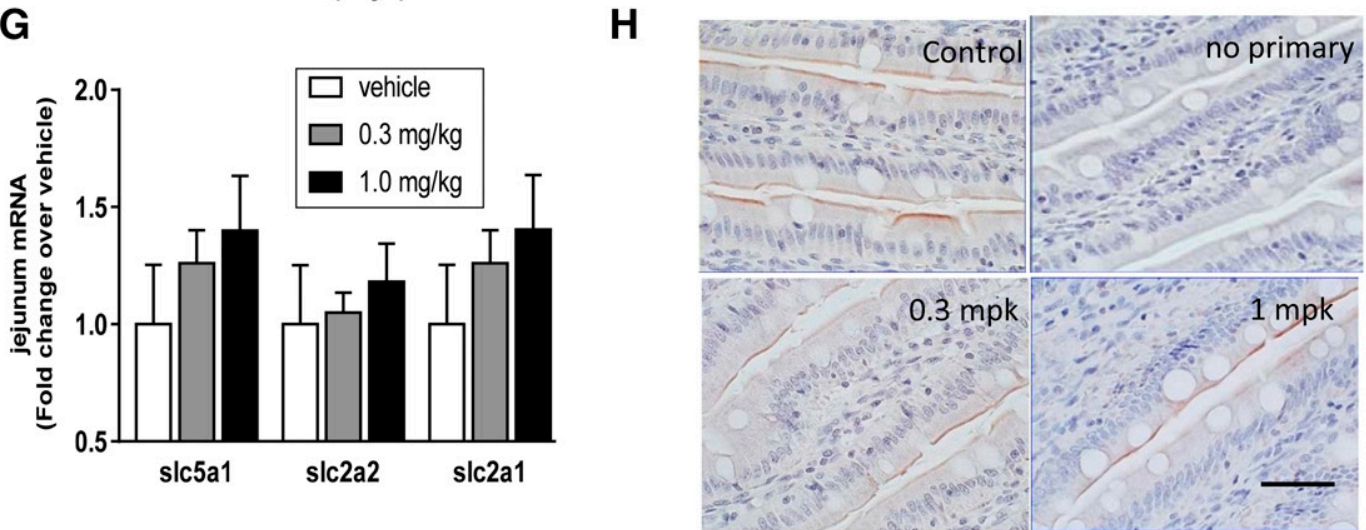

Fig. 5. SGLT1/2 inhibitor compound 8 given chronically to ZDF rats improved indices of hyperglycemia. (A) Compound 8 (squares, $0.3 \mathrm{mg} / \mathrm{kg}$; triangles, $1.0 \mathrm{mg} / \mathrm{kg}$ ) decreased fed blood glucose in a dose-related manner from day 0 to day 15 compared with elevated levels in vehicle-treated (circles). (B and C) On day 15, both doses (light and dark gray bars) compared with vehicle (white bar) decreased the AUC of glucose excursion after an oGTT after compound 8 was dosed 1 hour earlier $(\mathrm{B})$ and reduced circulating HbA1c $(\mathrm{C})$. (D and E) The higher dose of compound 8 increased total GLP-1 (D) and the gain in body weight at day 15 compared with vehicle (E). $* P<0.05$ (both doses compared with vehicle-treated mice); ${ }^{*} P<0.05(1.0 \mathrm{mg} / \mathrm{kg}$ dose compared with vehicle-treated mice); ${ }^{\#} P<0.05(1.0 \mathrm{mg} / \mathrm{kg}$ compared with $0.3 \mathrm{mg} / \mathrm{kg}$ treated rats by one-way or two-way analysis of variance). (F) Twenty-four hours after compound 8 administration $(1 \mathrm{mg} / \mathrm{kg}$ ), tissue levels were detected in intestinal mucosa and serosa/muscularis, as well as kidney. Bars indicate the following: white, intestinal luminal contents; stippled, mucosa; filled, serosa/muscularis externa; and hashed, kidney. (G) No effect of compound $8(0.3$ and $1.0 \mathrm{mg} / \mathrm{kg}$ ) relative to vehicle-treated jejunum mucosa mRNA levels encoding SGLT1 (slc5a1; black bar), GLUT1 (slc2a1; white bar), or GLUT2 (slc2a2; gray bar). (H) Representative digital images of SGLT1 immunohistochemical staining in brush border membrane. Scale bar, $100 \mu \mathrm{m}$. 

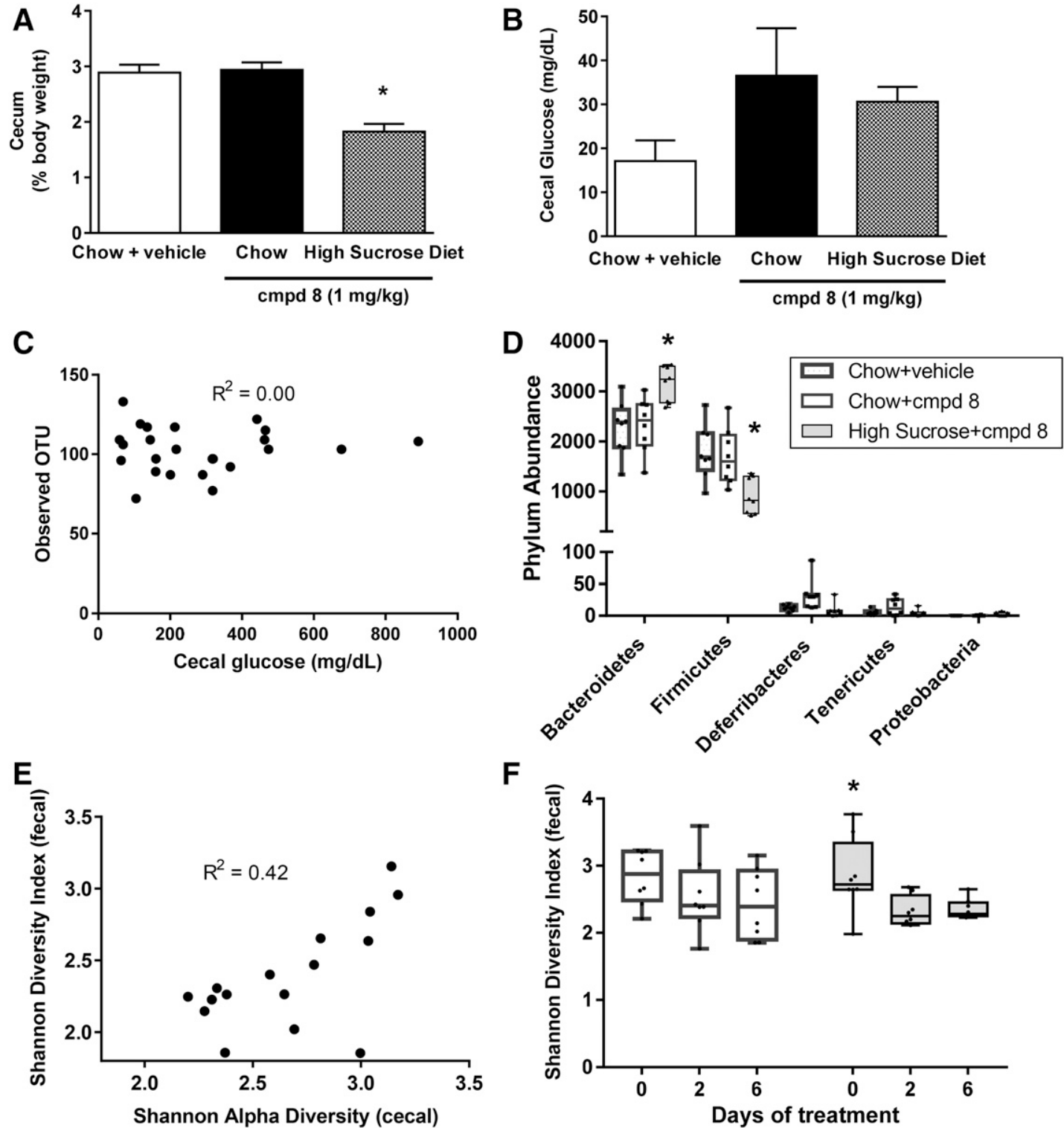

Fig. 6. Cecal glucose levels and microbial diversity were largely not affected in mice dosed compound 8 ( $1 \mathrm{mg} / \mathrm{kg})$ for 6 days unless mice were fed a highsucrose diet. (A) Gross cecal weight at termination was decreased in mice fed a high-sucrose diet and treated with compound 8 . $* P<0.05$ (compared with vehicle). (B) Although there was a tendency to higher cecal glucose levels in mice given compound 8 with chow (black bar) or a high-sucrose diet (checkered bar), individual variability resulted in no overall difference compared with chow-fed vehicle-treated mice. (C) At termination, cecal bacterial diversity (OTU) was not correlated with cecal glucose concentration in the same mice. (D) At termination, the cecal relative abundance of phyla was higher for Bacteroidetes and lower for Firmicutes in mice fed a high-sucrose diet with compound 8; there was no difference between chow-fed mice dosed with vehicle of compound 8. (E) At day 6 when mice were euthanised and cecal samples obtained microbiota diversity in fecal and cecal contents was highly correlated, as measured by the SDI. (F) Fecal SDI changed on day 2 after mice were switched to a high-sucrose diet with compound $8(P<0.05$; gray box and whisker plots) and was maintained to day 6, whereas the fecal SDI in compound 8-treated and chow-fed mice (white box and whisker plots) was similar throughout the study.

\section{Discussion}

The key findings are that potent SGLT1/2 dual inhibition by compound 8, more so than SGLT2-selective inhibition by dapagliflozin, reduced the oGTT glucose excursion and renal glucose thresholds. When given chronically to $\mathrm{ZDF}$ rats, compound 8 reduced $\mathrm{HbA1c}$ levels with increased circulating total GLP-1 as expected through intestinal mechanisms. This was not associated with obvious small intestinal adaptation, such as mucosal growth or villus length, or with compensatory changes in SGLT1 expression. In vivo compound 8 did not elevate colonic luminal glucose levels overall, potentially a consequence of small intestinal glucose "escaping" to the colon. Colonic glucose levels were not associated with altered bacterial population changes during the time frame studied, although there was an impact on the Firmicutes/Bacteroidetes ratio when compound 8 was combined with a high-sucrose diet. These points with be discussed in the context of the literature.

Glucose absorption from the small intestine could not be completely abolished in either slc5a $1^{-/-}$mice or after compound 8 treatment. Loss of expression was confirmed in jejunal mucosa from slc5a1 ${ }^{-/-}$mice and intermediate between WT and slc5 $\mathrm{a}^{-/-}$in slc5a1 ${ }^{+/-}$mice. Mice had similar baseline blood 
glucose levels but after an oGTT, the resulting ${ }^{13} \mathrm{C}$-glucose excursion was about $25 \%$ of WT levels. Interestingly, there was no genetic "titration" of glucose uptake in heterozygous mice, which had similar ${ }^{13} \mathrm{C}_{6}$-glucose uptake to WT mice. Paracellular uptake of glucose occurs by solvent drag through intercellular junctions that are dilated by perijunctional contractile proteins during fluid absorption induced by $\mathrm{Na}^{+}$ -coupled transport (Pappenheimer and Reiss, 1987; Nusrat et al., 2000). In isolated rat jejunal mucosa, compound 8 demonstrated functional SGLT1 inhibition by the reduction in glucose uptake, but only to a maximal $50 \%$ reduction. This is likely due to the absence of blood circulation, which helps provide the gradient for paracellular absorption during a high nutrient load that would otherwise overwhelm the SGLT1 $V_{\max }$. Terminal insulin levels in slc5a1 $1^{-/-}$mice were low compared with WT mice. However, $\operatorname{sglt}^{-1-}$ mice respond to oral glucose with increased circulating GLP-1 (Powell et al., 2013a), suggesting that fermentation to SCFA enhances GLP-1 release in these mice.

In rats given compound 8, postchallenge glucose excursion was reduced similar to a related SGLT1/2 inhibitor, compound 19 (Kuo et al., 2018). At higher doses, postchallenge blood glucose levels were reduced below baseline and to a greater extent than SGLT2-selective inhibitor dapagliflozin. This is presumably due to the reduction in glucose uptake combined with a reduced renal threshold for UGE. Whether this would translate to a risk for hypoglycemia in patients is unclear. In a phase III trial of patients with type 1 diabetes mellitus (ClinicalTrials.gov identifier NCT02531035), dual selective LX4211/sotagliflozin achieved $\mathrm{HbA1c}<7.0$ at week 24 but rates of diabetic ketoacidosis were higher than with placebo (Garg et al., 2017). Documented hypoglycemia (blood glucose $\leq 70 \mathrm{mg} / \mathrm{dl}$ ) was similar for the sotagliflozin and placebo groups, and only three patients (two in the sotagliflozin group and one in the placebo group) discontinued the treatment due to severe hypoglycemia (Garg et al., 2017).

Both compound 8 and SGLT2-selective dapagliflozin evoked similar UGE in SD rats, but blood glucose levels were lower for compound 8. Compound 8 achieved complete inhibition of renal glucose reabsorption at higher doses because $R_{G}$ values were approximately 0 . Increased expression of renal SGLT1 in patients with T2DM may develop as a compensatory mechanism to increase glucose reabsorption. Indeed, SGLT1 mRNA expression is 4-fold greater in kidney biopsies of patients with T2DM compared with controls (Norton et al., 2017). Therefore, an advantage of the dual SGLT1/2 inhibition in the kidneys of patients with diabetes may add to the efficacy of SGLT2 inhibitors. SGLT2-selective inhibitors lowered $\mathrm{RT}_{\mathrm{G}}$ by about $65 \%$ in humans (Devineni and Polidori, 2015) and had a fractional UGE of approximately 50\% (Abdul-Ghani et al., 2013), whereas phlorizin was shown to completely inhibit renal glucose reabsorption (Chasis et al., 1933). Therefore, dual inhibitors like compound 8 may give substantially more UGE than currently available SGLT2-selective inhibitors.

Obese mice fed an HFD did not show consistent changes in jejunum slc5a1 mRNA with a significant increase, decrease, or no change compared with lean controls in three similar cohorts tested. Obese diabetic Otsuka Long-Evans Tokushima fatty rats did have increased slc5a1 small intestinal expression (Fujita et al., 1998) and SGLT1 expression was increased in morbidly obese, nondiabetic humans (Nguyen et al., 2015). We did not assess whether a high-carbohydrate diet may consistently alter SGLT1 expression rather than HFD feeding. Rats offered high-starch diets had elevated SGLT1 expression (Honma et al., 2009) and switching from a CHOfree regimen to glucose in the diet increased SGLT1 mRNA (Miyamoto et al., 1993). However, transcriptional regulation of SGLT1 may be relatively less important in the context of obesity compared with post-transcriptional regulation. For example, SGLT1 protein rapidly transports to the apical membrane (Wright et al., 1998; Hines et al., 2000) and its activity varies diurnally to meet the fluctuating availability of glucose in mice (Fatima et al., 2009). On the other hand, compound 8 over 3 weeks in ZDF rats (albeit with less than 24-hour exposure) did not alter the level of expression of mRNA encoding SGLT1, GLUT1, or GLUT2 mRNA in the jejunum mucosa. Jejunum brush border membrane SGLT1 immunostaining was qualitatively similar in all groups with an unaltered villus/crypt ratio, suggesting no evidence of intestinal adaptation.

In ZDF rats, total GLP-1 in response to oGTT was increased by SGLT1 inhibition, which is consistent with other reports, (Zambrowicz et al., 2012, 2013a; Powell et al., 2014). This may seem counterintuitive, since L cells do not release GLP-1 in response to glucose in SGLT1 $1^{-/-}$mice (Gorboulev et al., 2012; Parker et al., 2012; Röder et al., 2014). Instead, the enhanced incretin effect is probably due to glucose metabolism to SCFAs in the large intestine (Powell et al., 2013b), which in turn activates release of GLP-1 through GPR 41/43 (FFAR3/2). SGLT2-selective inhibitors, such as canagliflozin, can also be associated with increased GLP-1 secretion due to a transient inhibition of SGLT1 at high local luminal concentrations (Polidori et al., 2013b). The enhancement of GLP-1 release by canagliflozin is due to the increase in SCFA in the distal intestine (Hira et al., 2017).

We reasoned that subcutaneous administration of compound 8 might minimize the inhibition of intestinal SGLT1 during an oGTT and enable comparison of UGE at equivalent blood glucose levels. However, compound 8 given systemically not only reduced UGE after an oGGT, as expected, but also inhibited blood glucose excursion. Compound 8 was detected in the mucosa of the small intestine after systemic administration, consistent with SGLT1 engagement. Furthermore, after oral compound 8 dosing, the tissue/plasma ratio increased over time, suggesting a longer duration of action in the gut than the circulating plasma levels and terminal half-life of approximately 2 hours would indicate. Interestingly, the colonic luminal contents had increased compound 8 relative to the plasma levels 6 hours after subcutaneous dosing. This would be consistent with excretion of small intestinal brush border SGLT1-bound (or free) compound 8 from the systemic circulation that was carried by GI transit into the colon. Combined, these data suggest that compound 8 reaches the intestinal brush border rapidly after parenteral administration and inhibits SGLT1 glucose uptake for longer than the circulating half-life. SGLT1 inhibition in the small intestine is the primary mechanism for decreased blood glucose in this study; at the higher doses, glucose excursion was completely abolished, suggesting complete inhibition of uptake. In rodents, an increase in GLP-1 occurred after gut-restricted SGLT1-selective inhibitors (Powell et al., 2017) as well as diarrhea. The latter was decreased by pretreatment with resistant starch, to select for bacterial species that ferment glucose (Powell et al., 2017). Therefore, ingested glucose 
"escapes" into the large intestine, where it would be rapidly fermented as an optimal energy source for colonic bacteria. An increase in the residual glucose content has been observed in the GI tract of rats treated with the SGLT1 inhibitor KGA2727 (Shibazaki et al., 2012) or LX4211/sotagliflozin (Powell et al., 2014). In healthy humans, it is normal for some digestible carbohydrate to reach the colon (Bond et al., 1980; Anderson et al., 1981; Stephen et al., 1983). This so-called starch malabsorption amounts to about $10 \%-20 \%$ of a large starch meal, based on hydrogen breath tests. Mice had similar overall cecal glucose concentrations when dosed with compound 8 and fed chow or high-sucrose chow; however, we cannot rule that this was due to incomplete 24-hour SGLT1 inhibition. Cecal glucose levels were collected in the morning after overnight feeding. Interestingly, gross cecal weight was decreased in mice fed a high-sucrose diet and treated with compound 8 but cecal bacterial abundance (OTU) was not correlated with cecal glucose concentration. In mice fed a highsucrose diet with compound 8, there were higher Bacteroidetes and lower Firmicutes represented compared with mice fed chow with compound 8 and mice fed chow plus vehicle. Because cecal and fecal microbial diversity was highly correlated by SDI, we compared fecal bacterial diversity during the study. Fecal SDI changed on day 2 after mice were switched to a high-sucrose diet with compound 8 , but not after mice were fed compound 8 with chow. A high-sucrose diet induces obesity and changes in relative abundance of bacteria associated with metabolic disease, termed microbiota dysbiosis (e.g., Erysipelotrichaceae, Bacillus, Eubacterium, and Akkemansia spp.). Therefore, we conclude that increasing glucose by highsucrose diet in the presence of compound 8 can alter microbial populations; however, compound 8 alone resulted in no overall change in glucose levels or microbial dysbiosis.

This study demonstrates that a potent balanced dual SGLT1/2 inhibitor can improve glycemic control to a greater extent than SGLT2-selective compounds. This is not associated with obvious mucosal adaptation or microbial dysbiosis in these preclinical experiments. Whether this translates to sufficiently added benefit without GI adverse effects in patients with T2DM is unclear. The therapeutic margin for achieving additional glycemic benefit with a dual SGLT1/2 inhibitor compared with a potential risk for hypoglycemia due to the combined intestinal and kidney glycemic impact of SGLT1/2 inhibition is currently unknown.

\section{Acknowledgments}

The authors thank ABS Inc. and Jing Ma (Janssen, La Jolla, CA) for SGLT1 immunohistochemistry jejunum histology and morphometric analysis and Diversigen Inc. for analysis of microbiota. We also thank Bill Kintigh, Stephen O'Sullivan, Norman Huebert, and Corey Bender (Janssen, Spring House, PA) for tissue distribution studies.

\section{Authorship Contributions}

Participated in research design: Lenhard, Demarest, Liang, Hornby.

Conducted experiments: Du, Hinke, Cavanaugh, Wallace, Kirchner, Jennis.

Contributed new reagents or analytic tools: Kuo, Gaul.

Performed data analysis: Du, Hinke, Cavanaugh, Polidori, Jennis, Lang, Ajami.

Wrote or contributed to the writing of the manuscript: Du, Hinke, Polidori, Hornby.

\section{References}

Abdul-Ghani MA, DeFronzo RA, and Norton L (2013) Novel hypothesis to explain why SGLT2 inhibitors inhibit only $30-50 \%$ of filtered glucose load in humans. Diabetes 62:3324-3328.

Anderson IH, Levine AS, and Levitt MD (1981) Incomplete absorption of the carbohydrate in all-purpose wheat flour. $N$ Engl $J$ Med 304:891-892.

Balen D, Ljubojevic M, Breljak D, Brzica H, Zlender V, Koepsell H, and Sabolic I (2008) Revised immunolocalization of the Na+-D-glucose cotransporter SGLT1 in rat organs with an improved antibody. Am J Physiol Cell Physiol 295:C475-C489.

Bond JH, Currier BE, Buchwald H, and Levitt MD (1980) Colonic conservation of malabsorbed carbohydrate. Gastroenterology 78:444-447.

Caporaso JG, Lauber CL, Walters WA, Berg-Lyons D, Huntley J, Fierer N, Owens SM, Betley J, Fraser L, Bauer M, et al. (2012) Ultra-high-throughput microbial community analysis on the Illumina HiSeq and MiSeq platforms. ISME $J \mathbf{6}$ $1621-1624$

Caporaso JG, Lauber CL, Walters WA, Berg-Lyons D, Lozupone CA, Turnbaugh PJ, Fierer N, and Knight R (2011) Global patterns of 16S rRNA diversity at a depth of millions of sequences per sample. Proc Natl Acad Sci USA 108 (Suppl 1): $4516-4522$.

Cavin JB, Couvelard A, Lebtahi R, Ducroc R, Arapis K, Voitellier E, Cluzeaud F, Gillard L, Hourseau M, Mikail N, et al. (2016) Differences in alimentary glucose absorption and intestinal disposal of blood glucose after Roux-en-Y gastric bypass vs sleeve gastrectomy. Gastroenterology 150:454-464.

Chasis H, Jolliffe N, and Smith HW (1933) The action of phlorizin on the excretion of glucose, xylose, sucrose, creatinine and urea by man. J Clin Invest 12:1083-1090.

Chen J, Williams S, Ho S, Loraine H, Hagan D, Whaley JM, and Feder JN (2010) Quantitative PCR tissue expression profiling of the human SGLT2 gene and related family members. Diabetes Ther 1:57-92.

De Jonghe S, Proctor J, Vinken P, Feyen B, Wynant I, Marien D, Geys H, Mamidi RN, and Johnson MD (2014) Carcinogenicity in rats of the SGLT2 inhibitor canagliflozin. Chem Biol Interact 224:1-12.

Devineni D and Polidori D (2015) Clinical pharmacokinetic, pharmacodynamic, and drug-drug interaction profile of canagliflozin, a sodium-glucose co-transporter 2 inhibitor. Clin Pharmacokinet 54:1027-1041.

Dobbins RL, Greenway FL, Chen L, Liu Y, Breed SL, Andrews SM, Wald JA, Walker A, and Smith CD (2015) Selective sodium-dependent glucose transporter 1 inhibitors block glucose absorption and impair glucose-dependent insulinotropic peptide release. Am J Physiol Gastrointest Liver Physiol 308:G946-G954.

Fatima J, Iqbal CW, Houghton SG, Kasparek MS, Duenes JA, Zheng Y, and Sarr MG (2009) Hexose transporter expression and function in mouse small intestine: role of diurnal rhythm. J Gastrointest Surg 13:634-641.

Frankel WL, Zhang W, Afonso J, Klurfeld DM, Don SH, Laitin E, Deaton D, Furth EE, Pietra GG, Naji A, et al. (1993) Glutamine enhancement of structure and function in transplanted small intestine in the rat. JPEN J Parenter Enteral Nutr 17:47-55.

Fujita Y, Kojima H, Hidaka H, Fujimiya M, Kashiwagi A, and Kikkawa R (1998) Increased intestinal glucose absorption and postprandial hyperglycaemia at the early step of glucose intolerance in Otsuka Long-Evans Tokushima fatty rats. Diabetologia 41:1459-1466.

Fushimi N, Teranishi H, Shimizu K, Yonekubo S, Ohno K, Miyagi T, Itoh F, Shibazaki T, Tomae M, Ishikawa-Takemura Y, et al. (2013) Design, synthesis, and structure-activity relationships of a series of 4-benzyl-5-isopropyl-1H-pyrazol-3-yl $\beta$-D-glycopyranosides substituted with novel hydrophilic groups as highly potent inhibitors of sodium glucose co-transporter 1 (SGLT1). Bioorg Med Chem 21: $748-765$.

Garg SK, Henry RR, Banks P, Buse JB, Davies MJ, Fulcher GR, Pozzilli P, GestyPalmer D, Lapuerta P, Simó R, et al. (2017) Effects of sotagliflozin added to insulin in patients with type 1 diabetes. N Engl J Med 377:2337-2348.

Gorboulev V, Schürmann A, Vallon V, Kipp H, Jaschke A, Klessen D, Friedrich A Scherneck S, Rieg T, Cunard R, et al. (2012) Na(+)-D-glucose cotransporter SGLT1 is pivotal for intestinal glucose absorption and glucose-dependent incretin secretion. Diabetes 61:187-196.

Hines OJ, Whang EE, Bilchik AJ, Zinner MJ, Welton ML, Lane J, McFadden DW, and Ashley SW (2000) Role of $\mathrm{Na}+$-glucose cotransport in jejunal meal-induced absorption. Dig Dis Sci 45:1-6.

Hira T, Koga T, Sasaki K, and Hara H (2017) Canagliflozin potentiates GLP-1 secretion and lowers the peak of GIP secretion in rats fed a high-fat high-sucrose diet. Biochem Biophys Res Commun 492:161-165.

Honma K, Mochizuki K, and Goda T (2009) Inductions of histone H3 acetylation at lysine 9 on SGLT1 gene and its expression by feeding mice a high carbohydrate/fat ratio diet. Nutrition 25:40-44.

Inoue T, Takemura M, Fushimi N, Fujimori Y, Onozato T, Kurooka T, Asari T, Takeda H, Kobayashi M, Nishibe H, et al. (2017) Mizagliflozin, a novel selective SGLT1 inhibitor, exhibits potential in the amelioration of chronic constipation. Eur J Pharmacol 806:25-31.

Kuo GH, Gaul MD, Liang Y, Xu JZ, Du F, Hornby P, Xu G, Qi J, Wallace N, Lee S, et al. (2018) Synthesis and biological evaluation of benzocyclobutane-C-glycosides as potent and orally active SGLT1/SGLT2 dual inhibitors. Bioorg Med Chem Lett 28:1182-1187.

Lang W, Qi J, and Caldwell GW (2014) Drug, lipid, and acylcarnitine profiling using dried blood spot (DBS) technology in drug discovery, in Optimization in Drug Discovery: Methods in Pharmacology and Toxicology (Caldwell G and Yan Z eds) pp 461-475, Humana Press, Totowa, NJ.

Lapuerta P, Zambrowicz B, Strumph P, et al. (2015) Development of sotagliflozin, a dual sodium-dependent glucose transporter $1 / 2$ inhibitor. Diab Vasc Dis Res 12: $101-110$.

Lehmann A and Hornby PJ (2016) Intestinal SGLT1 in metabolic health and disease. Am J Physiol Gastrointest Liver Physiol 310:G887-G898.

Miyamoto K, Hase K, Takagi T, Fujii T, Taketani Y, Minami H, Oka T, and Nakabou Y (1993) Differential responses of intestinal glucose transporter mRNA transcripts to levels of dietary sugars. Biochem $J$ 295:211-215. 
Mudaliar S, Polidori D, Zambrowicz B, and Henry RR (2015) Sodium-glucose cotransporter inhibitors: effects on renal and intestinal glucose transport: from bench to bedside. Diabetes Care 38:2344-2353.

Nguyen NQ, Debreceni TL, Bambrick JE, Chia B, Wishart J, Deane AM, Rayner CK, Horowitz M, and Young RL (2015) Accelerated intestinal glucose absorption in morbidly obese humans: relationship to glucose transporters, incretin hormones, and glycemia. J Clin Endocrinol Metab 100:968-976.

Norton L, Shannon CE, Fourcaudot M, Hu C, Wang N, Ren W, Song J, Abdul-Ghani M, DeFronzo RA, Ren J, et al. (2017) Sodium-glucose co-transporter (SGLT) and glucose transporter (GLUT) expression in the kidney of type 2 diabetic subjects. Diabetes Obes Metab 19:1322-1326.

Nusrat A, Turner JR, and Madara JL (2000) Molecular physiology and pathophysiology of tight junctions. IV. Regulation of tight junctions by extracellular stimuli: nutrients, cytokines, and immune cells. Am J Physiol Gastrointest Liver Physiol 279:G851-G857.

Oguma T, Kuriyama C, Nakayama K, Matsushita Y, Hikida K, Tsuda-Tsukimoto M, Saito A, Arakawa K, Ueta K, Minami M, et al. (2016) Changes in glucose-induced plasma active glucagon-like peptide- 1 levels by co-administration of sodiumglucose cotransporter inhibitors with dipeptidyl peptidase-4 inhibitors in rodents. J Pharmacol Sci 132:255-261.

Pappenheimer JR and Reiss KZ (1987) Contribution of solvent drag through intercellular junctions to absorption of nutrients by the small intestine of the rat. $J$ Membr Biol 100:123-136.

Parker HE, Adriaenssens A, Rogers G, Richards P, Koepsell H, Reimann F, and Gribble FM (2012) Predominant role of active versus facilitative glucose transport for glucagon-like peptide-1 secretion. Diabetologia 55:2445-2455.

Pode-Shakked B, Reish O, Aktuglu-Zeybek C, Kesselman D, Dekel B, Bujanover Y, and Anikster Y (2014) Bitterness of glucose/galactose: novel mutations in the SLC5A1 gene. J Pediatr Gastroenterol Nutr 58:57-60.

Polidori D, Sha S, Ghosh A, Plum-Mörschel L, Heise T, and Rothenberg P (2013a) Validation of a novel method for determining the renal threshold for glucose excretion in untreated and canagliflozin-treated subjects with type 2 diabetes mellitus. J Clin Endocrinol Metab 98:E867-E871.

Polidori D, Sha S, Mudaliar S, Ciaraldi TP, Ghosh A, Vaccaro N, Farrell K, Roth enberg P, and Henry RR (2013b) Canagliflozin lowers postprandial glucose and insulin by delaying intestinal glucose absorption in addition to increasing urinary glucose excretion: results of a randomized, placebo-controlled study. Diabetes Care 36:2154-2161.

Powell DR, DaCosta CM, Gay J, Ding ZM, Smith M, Greer J, Doree D, Jeter-Jones S, Mseeh F, Rodriguez LA, et al. (2013a) Improved glycemic control in mice lacking Sglt1 and Sglt2. Am J Physiol Endocrinol Metab 304:E117-E130.

Powell DR, DaCosta CM, Smith M, Doree D, Harris A, Buhring L, Heydorn W, Nouraldeen A, Xiong W, Yalamanchili P, et al. (2014) Effect of LX4211 on glucose homeostasis and body composition in preclinical models. J Pharmacol Exp Ther 350:232-242.

Powell DR, Smith M, Greer J, Harris A, Zhao S, DaCosta C, Mseeh F, Shadoan MK Sands A, Zambrowicz B, et al. (2013b) LX4211 increases serum glucagon-like peptide 1 and peptide YY levels by reducing sodium/glucose cotransporter 1 (SGLT1)-mediated absorption of intestinal glucose. J Pharmacol Exp Ther $\mathbf{3 4 5}$ 250-259.
Powell DR, Smith MG, Doree DD, Harris AL, Greer J, DaCosta CM, Thompson A, Jeter-Jones S, Xiong W, Carson KG, et al. (2017) LX2761, a sodium/glucose cotransporter 1 inhibitor restricted to the intestine, improves glycemic control in mice. J Pharmacol Exp Ther 362:85-97.

Röder PV, Geillinger KE, Zietek TS, Thorens B, Koepsell H, and Daniel H (2014) The role of SGLT1 and GLUT2 in intestinal glucose transport and sensing. PLoS One $\mathbf{9}$ : e89977.

Sha S, Devineni D, Ghosh A, Polidori D, Chien S, Wexler D, Shalayda K, Demarest K, and Rothenberg P (2011) Canagliflozin, a novel inhibitor of sodium glucose co-transporter 2 , dose dependently reduces calculated renal threshold for glucose excretion and increases urinary glucose excretion in healthy subjects. Diabetes Obes Metab 13:669-672.

Shibazaki T, Tomae M, Ishikawa-Takemura Y, Fushimi N, Itoh F, Yamada M, and Isaji M (2012) KGA-2727, a novel selective inhibitor of a high-affinity sodium glucose cotransporter (SGLT1), exhibits antidiabetic efficacy in rodent models. $J$ Pharmacol Exp Ther 342:288-296.

Song P, Onishi A, Koepsell H, and Vallon V (2016) Sodium glucose cotransporter SGLT1 as a therapeutic target in diabetes mellitus. Expert Opin Ther Targets 20: 1109-1125.

Stephen AM, Haddad AC, and Phillips SF (1983) Passage of carbohydrate into the colon. Direct measurements in humans. Gastroenterology 85:589-595.

Vallaeys L, Van Biervliet S, De Bruyn G, Loeys B, Moring AS, Van Deynse E, and Cornette L (2013) Congenital glucose-galactose malabsorption: a novel deletion within the SLC5A1 gene. Eur $J$ Pediatr 172:409-411.

Wright EM, Loo DD, Panayotova-Heiermann M, Hirayama BA, Turk E, Eskandari S, and Lam JT (1998) Structure and function of the Na+/glucose cotransporter. Acta Physiol Scand Suppl 643:257-264.

Yoshikawa T, Inoue R, Matsumoto M, Yajima T, Ushida K, and Iwanaga T (2011) Comparative expression of hexose transporters (SGLT1, GLUT1, GLUT2 and GLUT5) throughout the mouse gastrointestinal tract. Histochem Cell Biol 135: 183-194.

Zambrowicz B, Ding ZM, Ogbaa I, Frazier K, Banks P, Turnage A, Freiman J, Smith M, Ruff D, Sands A, et al. (2013a) Effects of LX4211, a dual SGLT1/SGLT2 inhibitor, plus sitagliptin on postprandial active GLP-1 and glycemic control in type 2 diabetes. Clin Ther 35:273-285.

Zambrowicz B, Freiman J, Brown PM, Frazier KS, Turnage A, Bronner J, Ruff D, Shadoan M, Banks P, Mseeh F, et al. (2012) LX4211, a dual SGLT1/SGLT2 inhibitor, improved glycemic control in patients with type 2 diabetes in a randomized, placebo-controlled trial. Clin Pharmacol Ther 92:158-169.

Zambrowicz B, Ogbaa I, Frazier K, Banks P, Turnage A, Freiman J, Boehm KA, Ruff D, Powell D, and Sands A (2013b) Effects of LX4211, a dual sodium-dependent glucose cotransporters 1 and 2 inhibitor, on postprandial glucose, insulin, glucagon-like peptide 1 , and peptide tyrosine tyrosine in a dose-timing study in healthy subjects. Clin Ther 35:1162-1173.

Address correspondence to: Pamela J. Hornby, Cardiovascular and Metabolism Discovery, Janssen R\&D LLC, 1440 McKean Road, Bldg. SH422510-A, Spring House, PA 19477. E-mail: phornby@its.jnj.com 